\title{
High expression of ACTL8 is poor prognosis and accelerates cell progression in head and neck squamous cell carcinoma
}

\author{
BO LI, JIE ZHU and LEI MENG
}

Department of Neurosurgery, Jinan Central Hospital Affiliated to Shandong University, Jinan, Shandong 250013, P.R. China

Received January 2, 2018; Accepted October 11, 2018

DOI: $10.3892 / \mathrm{mmr} .2018 .9716$

\begin{abstract}
Actin-like protein 8 (ACTL8) is a member of the cancer-testis antigens (CTA) family, which is mainly localized in the cytoplasm and generally expressed in the testis. The association between ACTL8 and various types of cancer, including glioblastoma and breast cancer, has previously been demonstrated. However, whether ACTL8 is involved in the development of head and neck squamous cell carcinoma (HNSCC) remains unknown. In the present study, the expression of ACTL8 in patients with HNSCC was analyzed in The Cancer Genome Atlas (TCGA) dataset, clinical tissues and cell lines. Correlations between the expression levels of ACTL8 and HNSCC clinical outcomes were analyzed with the Kaplan-Meier method and the Cox proportional hazards model. Cell Counting Kit-8, plate colony formation and Transwell assays were used to assess the effects of ACTL8 interference on the proliferation, migration and invasion of HNSCC PCI-13 cells. Reverse transcription-quantitative polymerase chain reaction and western blotting were used to evaluate the expression levels of ACTL8 in PCI-13 cells. Furthermore, alterations in the expression levels of key proteins in the phosphatidylinositol-4,5-bisphosphate 3-kinase (PI3K)/RAC- $\alpha$ protein kinase $\mathrm{B}$ (AKT) signaling pathway were determined by western blotting. Increased expression of ACTL8 in HNSCC was observed in TCGA dataset, cancerous tissue samples and HNSCC cell line. Cox regression analysis indicated that ACTL8 expression could be regarded as an independent prognostic factor for HNSCC, since increased expression of ACTL8 was associated with a poor prognosis. Knocking down ACTL8 markedly inhibited the proliferation, invasion and migration of PCI-13 cells. Additionally, activation of the PI3K/AKT signaling pathway was suppressed by reduced expression levels of certain key proteins in this pathway. The present data indicate that ACTL8 serves a role in the progression and clinical prognosis
\end{abstract}

Correspondence to: Dr Lei Meng, Department of Neurosurgery, Jinan Central Hospital Affiliated to Shandong University, 105 Jiefang Road, Jinan, Shandong 250013, P.R. China

E-mail: mengleiyutian75@126.com

Key words: head and neck squamous cell carcinoma, prognosis, cell proliferation, ACTL8 of HNSCC. Therefore, ACTL8 may be a potential prognostic marker and novel therapeutic target for HNSCC.

\section{Introduction}

Head and neck squamous cell carcinoma (HNSCC) is essentially a heterogeneous disease, usually resulting from the mucosal lining of the upper respiratory tract, including oral cavity, nasal cavity and throat. HNSCC is ranked sixth in the leading cause of cancer-associated fatalities worldwide. It is characterized by complex anatomy, regional diffusion, distant metastasis and high recurrence. HNSCC can induce the production of cytokines and growth factors that participate in the processes of gene expression, cell growth, survival and chemosensitivity $(1,2)$. Over the past few decades, although the diagnostic techniques and treatment methods have made great progress, the morbidity and mortality rates of HNSCC patients remain high (3). The development of novel molecular biology investigation techniques contributes to identify the molecules that serve an inhibitory role or facilitating role in tumors. Targeting these molecules has become an effective strategy for the treatment of cancer and may ultimately improve the disease-free survival rates of cancer patients.

Cancer-testis antigens (CTAs) are a class of tumor-associated antigens that contains more than 200 members, characterized by specific expression in placental tissue and in a variety of tumor tissues and testes, but expressed at a low level or not expressed in other normal tissues (4). Previously, certain CTAs have been studied as target antigens in clinical trials and demonstrated available therapeutic effect on the treatment of glioblastoma, particularly for melanoma associated antigen (MAGEA)1 and NY-ESO1 (5). Additionally, frequent expression of specific CTA genes in HNSCC has been studied. For example, Atanackovic et al (6) have reported that the expression of MAGEA3 and SSX1 in HNSCC was 72 and $45 \%$, respectively. However, the roles of CTA genes in HNSCC remain unknown. Actin-like protein 8 (ACTL8) is a member of the CTA family and contains 366 amino acids. ACTL8 is also known as CT57, as it is the 57th member of the CTA family (http://www.CTA.Incc.br). The ACTL8 gene was reported to be highly expressed in glioblastoma, whereas it was expressed at a low level in the bladder, pancreas, thymus and colon tissues, and not expressed in normal brain tissues (7). Yao et al (8) have suggested that the expression of ACTL8 gene in breast cancer was also upregulated. ACTL8 
gene is located on chromosome $1 \mathrm{p} 36.13$ and the aberrations of chromosome 1 occur frequently in head and neck cancer (9). However, to date, there is little direct evidence of the role of ACTL8 in head and neck cancer.

In order to investigate the potential role of ACTL8, the present study investigated the expression levels and clinical significance of ACTL8 in HNSCC. Furthermore, the effects of ACTL8 on the proliferation, invasion and migration of HNSCC PCI-13 cells were determined by knockdown of ACTL8. In addition, alterations in the expression levels of proteins involved in the phosphatidylinositol-4,5-bisphosphate 3-kinase (PI3K)/protein kinase B (AKT) signaling pathway were detected upon transfection of PCI-13 cells with ACTL8 small interfering RNA (siRNA). The results indicated that ACTL8 may serve an important role in HNSCC progression and could be considered a significant prognostic marker and therapeutic target for HNSCC.

\section{Materials and methods}

Patients. A total of 110 patients of HNSCC, who had received curative surgery at the Department of Otolaryngology-Head and Neck Surgery in Jinan Central Hospital Affiliated to Shandong University (Jinan, China) were selected by reviewing the medical records between January 2002 and December 2014. Furthermore, 110 adjacent normal tissues taken from the corresponding patients were selected. Notably, no radiotherapy and chemotherapy were performed for all samples prior to surgery. Half specimens were imbedded with paraffin following fixation with $10 \%$ formalin at room temperature for $48 \mathrm{~h}$ and protected from light at room temperature. The other half number of specimens were used to detect mRNA expression levels. The clinical features including age, gender, pathological-stage and tumor status are presented in Table I. This study obtained the informed consent of all patients and was approved by the Jinan Central Hospital Affiliated to Shandong University Medical Ethics Committee.

In the present study, the gene expression profile information for patients with HNSCC was obtained from TCGA data portal (cancergenome.nih.gov). A total of 502 samples with HNSCC as disease group and 44 healthy samples as a control group were selected.

Cell culture. Human HNSCC cell line PCI-13 was purchased from the Chinese Academy of Medical Sciences cell bank (Shanghai, China). Normal oral cells of primary gingival keratinocytes, which were used as a control were obtained from the American Type Culture Collection, (Manassas, VA, USA; PCS-200-014 ${ }^{\mathrm{TM}}$ ). Cells were cultured in RPMI1640 (Gibco; Thermo Fisher Scientific, Inc., Waltham, MA, USA) medium at a humidified chamber of $37^{\circ} \mathrm{C}$ containing $5 \% \mathrm{CO}_{2}$. In addition, $10 \%$ heat-inactivated fetal bovine serum (FBS, Thermo Fisher Scientific, Inc.), $100 \mu \mathrm{g} / \mathrm{ml}$ streptomycin and $100 \mathrm{U} / \mathrm{ml}$ penicillin (Thermo Fisher Scientific, Inc.) were supplemented in the medium.

RNA extraction and reverse transcription-quantitative polymerase chain reaction $(R T-q P C R)$ detection. Total RNA was separated from PCI-13 cells using TRIzol reagent
(Invitrogen; Thermo Fisher Scientific, Inc.) according to the manufacturer's protocol. Reverse transcription was conducted on $3 \mu \mathrm{g}$ of total RNA per sample to generate cDNA with a RevertAid First Strand cDNA Synthesis kit (Fermentas, Thermo Fisher Scientific, Inc.). The obtained cDNAs were used in amplification, analysis and quantification of PCR. Probes were amplified by RT-PCR with specific primers. The primer sequences of ACTL 8 mRNA were designed and synthesized as follows: 5'-GCCACGTGCTCACAGAGT AG-3' (forward) and 5'-CTCAGCTGCACACTGCAAAC-3' (reverse). GAPDH was used as an internal control. The primer sequences of GAPDH were as follows: Forward, 5'-GGAGCG AGATCCCTCCAAAAT-3' and reverse, 5'-GGCTGTTGT CATACTTCTCATGG-3' (Table II). The PCR cycle condition was set as follows: $95^{\circ} \mathrm{C}$ for $5 \mathrm{~min}$, followed by 40 cycles of $95^{\circ} \mathrm{C}$ for $30 \mathrm{sec}, 60^{\circ} \mathrm{C}$ for $45 \mathrm{sec}, 72^{\circ} \mathrm{C}$ for $30 \mathrm{~min}$. For the RT-qPCR analysis, comparative $\mathrm{C}_{\mathrm{q}}$ method ( $\mathrm{DDC}_{\mathrm{q}}$ ) (10) was performed to compute the expression levels. All experiments were independently repeated three times.

Western blot assay. The cancer samples and cell line were lysed in RIPA lysis buffer (Beyotime Institute of Biotechnology, Nantong, China). Lysates were collected by centrifugation $13,400 \times \mathrm{g}$ at $4^{\circ} \mathrm{C}$ for $20 \mathrm{~min}$. The concentration of proteins was detected using bicinchoninic acid Protein Assay kit (CWBiotech Co., Ltd., Beijing, China) according to the manufacturer's protocol. Subsequently, equal amounts $(20 \mu \mathrm{g})$ of each sample were placed on a $10 \%$ polyacrylamide gel, isolated using $12 \%$ SDS-PAGE and then the samples were transferred onto a polyvinylidene fluoride membranes (EMD Millipore, Billerica, MA, USA). Furthermore, the membrane was blocked in TBS with 5\% non-fat dry milk at room temperature for $1 \mathrm{~h}$. Finally, western blot analysis was conducted by incubating the membrane with rabbit anti-human ACTL8 antibody (anti-ACTL8; 1:1,000; cat. no. sc-377372; Santa Cruz Biotechnology, Inc., Dallas, TX, USA), PI3K (1:1,000; cat. no. 4255), AKT (1:1,000; cat. no. 9272), P-70S6K (1:1,000; cat. no. 9202) and the phosphorylation antibody p-PI3K (1:1,000; cat. no. 4228), p-AKT (1:1,000; cat. no. 9271), p-p70S6K (1:1,000; cat. no. 9204; CST Biological Reagents Co., Ltd.) at $4^{\circ} \mathrm{C}$ overnight and incubated with secondary antibody anti-rabbit HRP (1:5,000; cat. no. 7074; CST Biological Reagents Co., Ltd.) at room temperature for $2 \mathrm{~h}$. GAPDH (1:2,000; cat. no. 5174) was used as control. Consequently, all blot analysis was conducted using a Pierce ${ }^{\mathrm{TM}}$ ECL Western Blotting Substrate kit (Thermo Fisher Scientific, Inc.).

Cell transfection. PCI-13 cells were placed in 6-well plates $\left(1 \times 10^{5}\right.$ cells/well) and cultured using serum-free medium when the density reached to $80 \%$. A total of $125 \mu 1$ opti-MEM medium (Thermo Fisher Scientific, Inc.) was used to mix the ACTL8 siRNA (si-ACTL8: 5'-GAGTCTCTTTAAGGAAGATTGCGAT-3') or negative control siRNA (5'-TAGCGTTAGAAGGAATTTCTC TGAG-3') at $50 \mathrm{nM}$. A total of $3.75 \mu$ l Lipofectamine-2000 (Thermo Fisher Scientific, Inc.) was extracted to mix with another $125 \mu \mathrm{l}$ opti-MEM medium and then was incubated for $5 \mathrm{~min}$. Subsequently, the mixture was re-seeded to a 6 -well plate after reaching $60-80 \%$ confluency and cultured at $37^{\circ} \mathrm{C}$ 
Table I. Correlation between clinicopathological parameters and ACTL8 expression in head and neck squamous cell carcinoma $(n=110)$.

\begin{tabular}{|c|c|c|c|}
\hline \multirow[b]{2}{*}{ Characteristics } & \multicolumn{2}{|c|}{$\begin{array}{l}\text { Expression } \\
\text { of ACTL8 }\end{array}$} & \multirow[b]{2}{*}{ P-value } \\
\hline & Low & High & \\
\hline Age & & & 0.699 \\
\hline$<60$ & 22 & 24 & \\
\hline$\geq 60$ & 33 & 31 & \\
\hline Sex & & & 0.152 \\
\hline Female & 21 & 14 & \\
\hline Male & 34 & 41 & \\
\hline Pathological-stage & & & 0.251 \\
\hline $\mathrm{I}+\mathrm{II}$ & 28 & 22 & \\
\hline III+IV & 27 & 33 & \\
\hline Tumor status & & & 0.702 \\
\hline $\mathrm{T} 1+\mathrm{T} 2$ & 30 & 28 & \\
\hline $\mathrm{T} 3+\mathrm{T} 4$ & 25 & 27 & \\
\hline Node metastasis & & & $0.002^{\mathrm{a}}$ \\
\hline N0 & 49 & 35 & \\
\hline N1 & 6 & 20 & \\
\hline Distant metastasis & & & 1.000 \\
\hline M0 & 54 & 53 & \\
\hline M1 & 1 & 2 & \\
\hline Grade & & & $0.021^{\mathrm{a}}$ \\
\hline $\mathrm{G} 1+\mathrm{G} 2$ & 48 & 38 & \\
\hline $\mathrm{G} 3+\mathrm{G} 4$ & 7 & 17 & \\
\hline
\end{tabular}

${ }^{\mathrm{a}} \mathrm{P}<0.05$ suggested significant comparison between Characteristics and Expression of ACTL8. ACTL8, actin-like protein 8.

for 3-5 days. Finally, the transfected cells were extracted for subsequent analysis.

Cell proliferation. PCI-13 cells were digested and counted following transfection for $72 \mathrm{~h}$ to prepare cell suspensions. A total of $100 \mu \mathrm{l}$ the cell suspension was extracted and seeded to a 96 -well plate at a density of $10^{3}$ cell/well, then placing in a carbon dioxide incubator for routine cultivation. Cell Counting Kit-8 (CCK-8; Beijing Solarbio Science \& Technology Co., Ltd., Beijing, China) was employed to measure the proliferation of PCI-13 cells, the results were detected at $0,24,48,72$ and $96 \mathrm{~h}$, respectively. Microplate reader at $450 \mathrm{~nm}$ was conducted to detect the absorbance of the wells in the plate.

Plate colony formation assay. Stably transfected PCI-13 cells were placed into a 6-well plate at a density of 500 cell/well in triplicate and maintained in DMEM containing 10\% FBS for cultivation. Following incubation for 12 days at $37^{\circ} \mathrm{C}$ in a humid chamber, the cells were washed with PBS and fixed using $4 \%$ paraformaldehyde for $30 \mathrm{~min}$ at room temperature. Subsequently, the fixative was removed and cells were stained with $0.1 \%$ crystal violet at room temperature for $30 \mathrm{~min}$. Finally, after the residual staining solution was rinsed with running water, the size and number of clones were counted under a light microscope.

Cell invasion and migration assays. For the cell invasion assay, $100 \mu 1$ Matrigel (serum-free medium diluted 1:6) dissolved overnight was added to the upper chamber of transwell chamber with a 24-well plate, which then was shaken and placed in carbon dioxide incubator for 4-6 h. After the addition of gelatin, $500 \mu \mathrm{l}$ of serum-free medium was added to the lower chamber and maintained for $30 \mathrm{~min}$ to hydrate the basement membrane. Cells following $24 \mathrm{~h}$ of transfection were used to prepare cell suspensions with serum-free medium and extracting $100 \mu \mathrm{l}$ cell suspension ( $1 \times 10^{5}$ cells) to add to the upper chamber and $500 \mu \mathrm{l}$ complete medium was added to the lower chamber. After overnight incubation, residual cells in the upper chamber were removed with a cotton-tipped swab. Cells penetrating the bottom chamber from the membrane were fixed in $4 \%$ paraformaldehyde at room temperature for $15 \mathrm{~min}$ and stained with $0.1 \%$ crystal violet at room temperature for $30 \mathrm{~min}$. A total of five fields of view were randomly selected to count the number of cells using a light microscope. The procedures in the migration assay is similar to the invasion assays. The difference is that there is no matrigel precoating in the transwell chamber for the migration assay.

Statistical analysis. Kaplan-Meier method was used to analyze the survival rate of patients with HNSCC. Furthermore, a log-rank test was performed to compute the difference between survival-associated curves. Cox proportional hazard regression models were applied to establish univariate and multivariate survival analysis for evaluating the independent prognostic factors. GraphPad Prism 5 (GraphPad Software, Inc., La Jolla, CA, USA) and SPSS 22.0 (IBM, Corps., Armonk, NY USA) were employed to conduct the statistical analysis. Chi-square test was performed to evaluate the clinicopathological features (Table I) and survival status (Fig. 2) of ACTL8 in HNSCC patients. A student's t-test (for two group samples) and one-way analysis of variance followed by Dunnett-t post-hoc test (for multiple group samples) were performed to compare the normally distributed data. All data in this study were expressed as the mean \pm standard deviation. $\mathrm{P}<0.05$ was considered to indicate a statistically significant difference.

\section{Results}

High expression of ACTL8 in HNSCC is associated with a poor prognosis. To identify the expression level of ACTL8 in HNSCC, expression of ACTL8 was analyzed in TCGA datasets and the collected HNSCC patients. Fig. 1A demonstrated the expression in normal and HNSCC samples, high expression was demonstrated in the HNSCC patients compared with the control group $\left(\mathrm{P}=5.86 \times 10^{-12}\right)$. Notably, compared to the expression levels in the normal mucosa group, ACTL8 in clinical samples of HNSCC was also highly expressed (<0.0001; Fig. 1B). Pearson's chi-squared test was applied to assess the correlation between ACTL8 expression and clinicopathological features. It is demonstrated in Table I that there is a significant association between ACTL8 expression with node metastasis $(\mathrm{P}=0.002)$ 
Table II. Primers sequences used.

\begin{tabular}{llr} 
Gene & \multicolumn{1}{c}{ Primer sequence } & PCR cycle condition \\
\hline ACTL8 & Forward: 5'-GCCACGTGCTCACAGAGTAG-3' & $95^{\circ} \mathrm{C} \mathrm{5} \mathrm{sec}$ \\
& Reverse: 5'-CTCAGCTGCACACTGCAAAC-3' & $95^{\circ} \mathrm{C} \mathrm{30} \mathrm{sec}$ \\
GAPDH & Forward: 5'-GGAGCGAGATCCCTCCAAAAT-3' & $60^{\circ} \mathrm{C} 45 \mathrm{sec}$ \\
& Reverse: 5'-GGCTGTTGTCATACTTCTCATGG-3' & $72^{\circ} \mathrm{C} \mathrm{30} \mathrm{sec}$
\end{tabular}

PCR, polymerase chain reaction; ACTL8, actin-like protein 8.
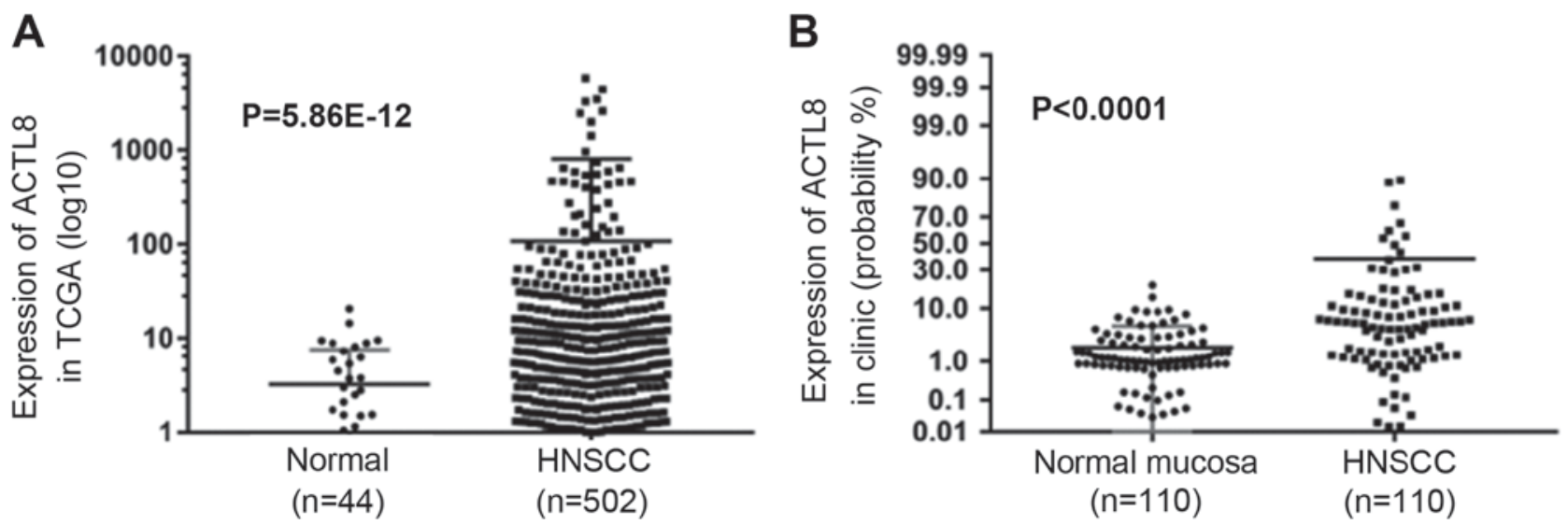

Figure 1. Relative expression of ACTL8 in HNSCC patients. (A) Analyzing the data from TCGA datasets, the ACTL8 expression in HNSCC tissues significantly increased compared with normal tissues. (B) The ACTL8 expression level in HNSCC tissues was markedly upregulated compared with the normal mucosa group. Data was presented as the mean \pm standard deviation. HNSCC, head and neck squamous cell carcinoma; ACTL8, actin-like protein 8; TCGA, The Cancer Genome Atlas.

and pathological grade $(\mathrm{P}=0.021)$. Whereas, the correlation between ACTL8 expression with other clinical parameters was not demonstrated (all $\mathrm{P}>0.05$ ).

To further confirm the potential associations between ACTL8 expression and prognosis of HNSCC patients, the associations between ACTL8 expression and clinical outcomes were measured. The Kaplan-Meier method using a log-rank test was conducted to measure the association between ACTL8 expression and survival status. The results in Fig. 2 demonstrated that the survival rates of HNSCC patients between high ACTL8 expression and low ACTL8 expression groups were significantly different, suggesting that HNSCC patients with ACTL8 high expression have a poor prognosis $\left(\chi^{2}=6.512, P=0.011\right)$. The univariate analysis was performed to investigate the prognostic factors. As presented in Table III, the ACTL8 expression $(\mathrm{P}=0.013)$, pathological-stage $(\mathrm{P}<0.0001)$, tumor status $(\mathrm{P}<0.0001)$, distant metastasis $(\mathrm{P}<0.0001)$ and node metastasis $(\mathrm{P}=0.008)$ were all the prognostic factors. Therefore, to further confirm an independent risk factor, multivariate analysis was conducted. Consequently, it was demonstrated that ACTL8 expression $(\mathrm{P}=0.029)$ and distant metastasis could be considered as an independent risk factor for overall survival in patients with HNSCC.

Effect of ACTL8 gene on HNSCC PCI-13 cells. RT-qPCR method results in Fig. 3A demonstrated that ACTL8 expression in PCI-13 cells was significantly increased compared with the normal oral mucosa group $(\mathrm{P}<0.01)$. To further confirm

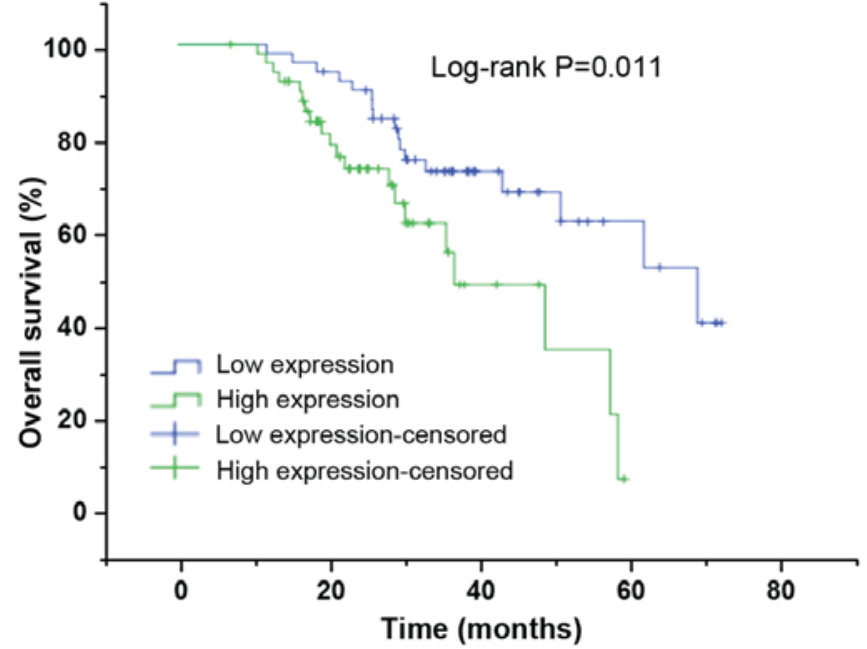

Figure 2. Kaplan-Meier curves demonstrate overall survival of head and neck squamous cell carcinoma patients with high ACTL8 expression $(n=55)$ vs. low ACTL8 expression $(n=55 ; P=0.011)$, using a long-rank test. ACTL8, actin-like protein 8 .

the function of ACTL8 in HNSCC PCI-13 cells, the ACTL8 siRNA was transfected into the HNSCC PCI-13 cell line. Following $72 \mathrm{~h}$ transfection, total RNA and proteins were extracted for performing RT-qPCR and western blot assays. The results in Fig. 3B demonstrated that there was no statistical difference between the blank control and interference control. 
Table III. Univariate and multivariate survival analysis for head and neck squamous cell carcinoma patients.

\begin{tabular}{|c|c|c|c|c|c|c|}
\hline \multirow[b]{2}{*}{ Variables } & \multicolumn{3}{|c|}{ Univariate analysis } & \multicolumn{3}{|c|}{ Multivariate analysis } \\
\hline & P-value & HR & $95 \% \mathrm{CI}$ & P-value & HR & $95 \% \mathrm{CI}$ \\
\hline ACTL8 expression (high/low) & $0.013^{\mathrm{a}}$ & 2.370 & $1.199-4.684$ & $0.029^{\mathrm{a}}$ & 2.277 & $1.090-4.757$ \\
\hline Pathological-stage (I+II/III+IV) & $0.000^{\mathrm{a}}$ & 4.725 & $2.218-10.065$ & 0.718 & 1.513 & $0.159-14.367$ \\
\hline Tumor status $(\mathrm{T} 1+\mathrm{T} 2 / \mathrm{T} 3+\mathrm{T} 4)$ & $0.000^{\mathrm{a}}$ & 4.824 & $2.329-9.990$ & 0.290 & 3.111 & $0.381-25.417$ \\
\hline Distant metastasis (M0/M1) & $0.000^{\mathrm{a}}$ & 12.490 & $3.359-46.446$ & $0.011^{\mathrm{a}}$ & 6.613 & $1.538-28.442$ \\
\hline Node metastasis $(\mathrm{N} / \mathrm{N} 1+\mathrm{N} 2+\mathrm{N} 3)$ & $0.008^{\mathrm{a}}$ & 2.771 & $1.309-5.864$ & 0.824 & 0.895 & $0.339-2.365$ \\
\hline
\end{tabular}

${ }^{a} \mathrm{P}<0.05$ suggested significant comparison between variables and overall survival. HR, hazard ratio; CI, confidence interval; ACTL8, actin-like protein 8 .
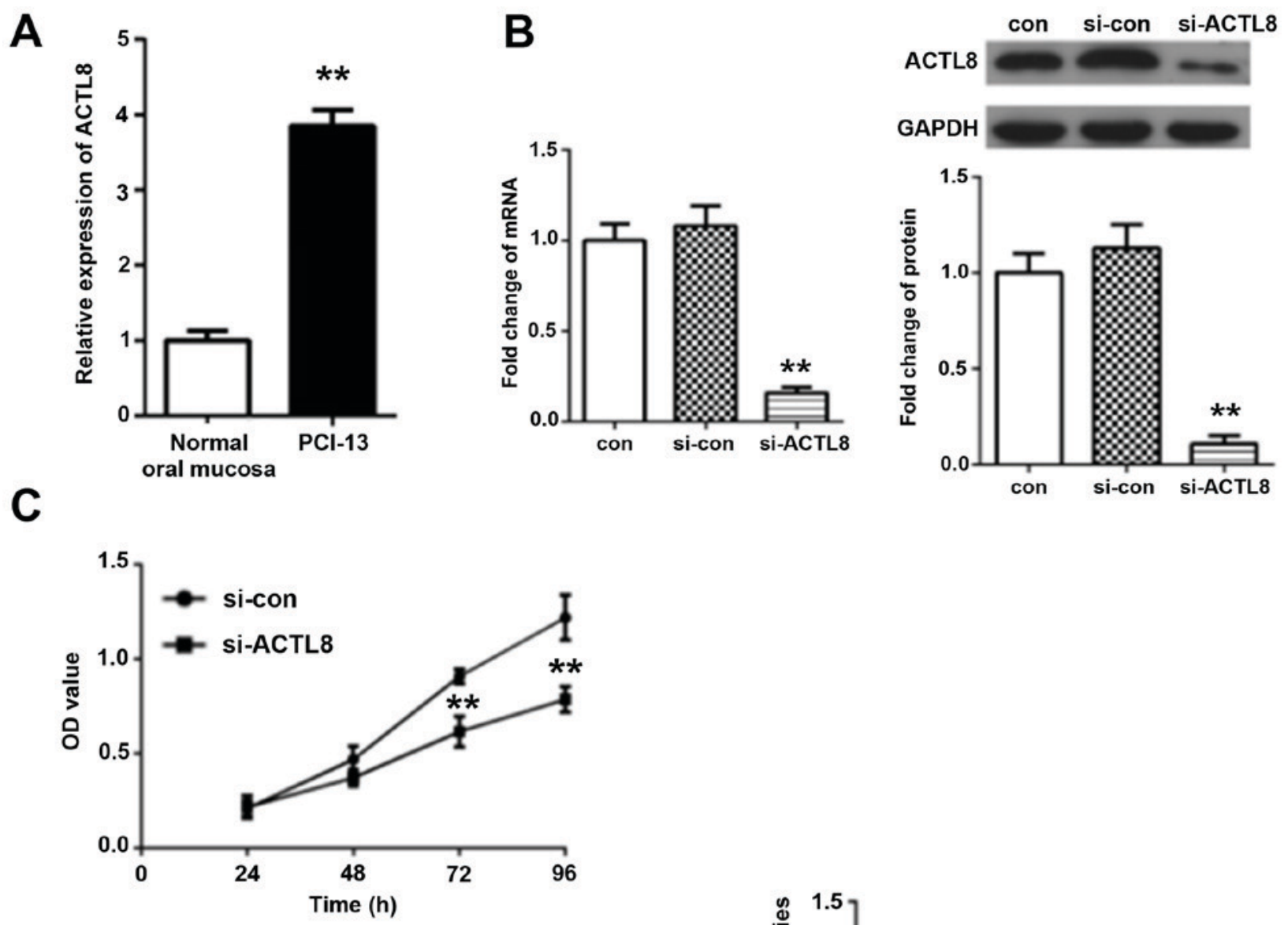

D
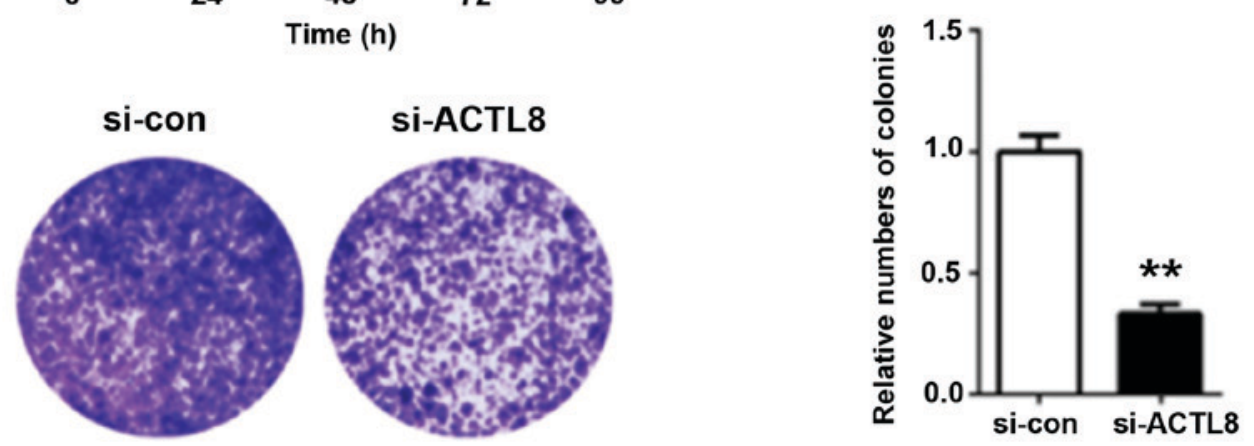

Figure 3. Effect of ACTL8 on the proliferation of HNSCC PCI-13 cells in vitro. (A) The relative expression of ACTL8 in PCI-13 cells vs. in the normal oral mucosa group. (B) Western blot assay and reverse transcription-quantitative polymerase chain reaction detection were used to evaluate the expression levels of mRNA and protein of ACTL8 following ACTL8 siRNA treatment. Particularly, no significant difference was demonstrated between the blank control (con) and interference control (si-con). (C) At $72 \mathrm{~h}$ following ACTL8 siRNA transfection, the viability of PCI-13 cells were measured by a Cell Counting Kit-8 assay by comparing with the si-con group. (D) Plate colony formation assay was conducted to confirm the colony formation rate of PCI-13 cells in si-ACTL8 group and si-con group. Data are presented as the mean \pm standard deviation. ${ }^{* *} \mathrm{P}<0.01$ vs. si-con. ACTL8, actin-like protein 8; si-ATCL8, small interfering; HNSCC, head and neck squamous cell carcinoma; con, control; OD, optical density. 

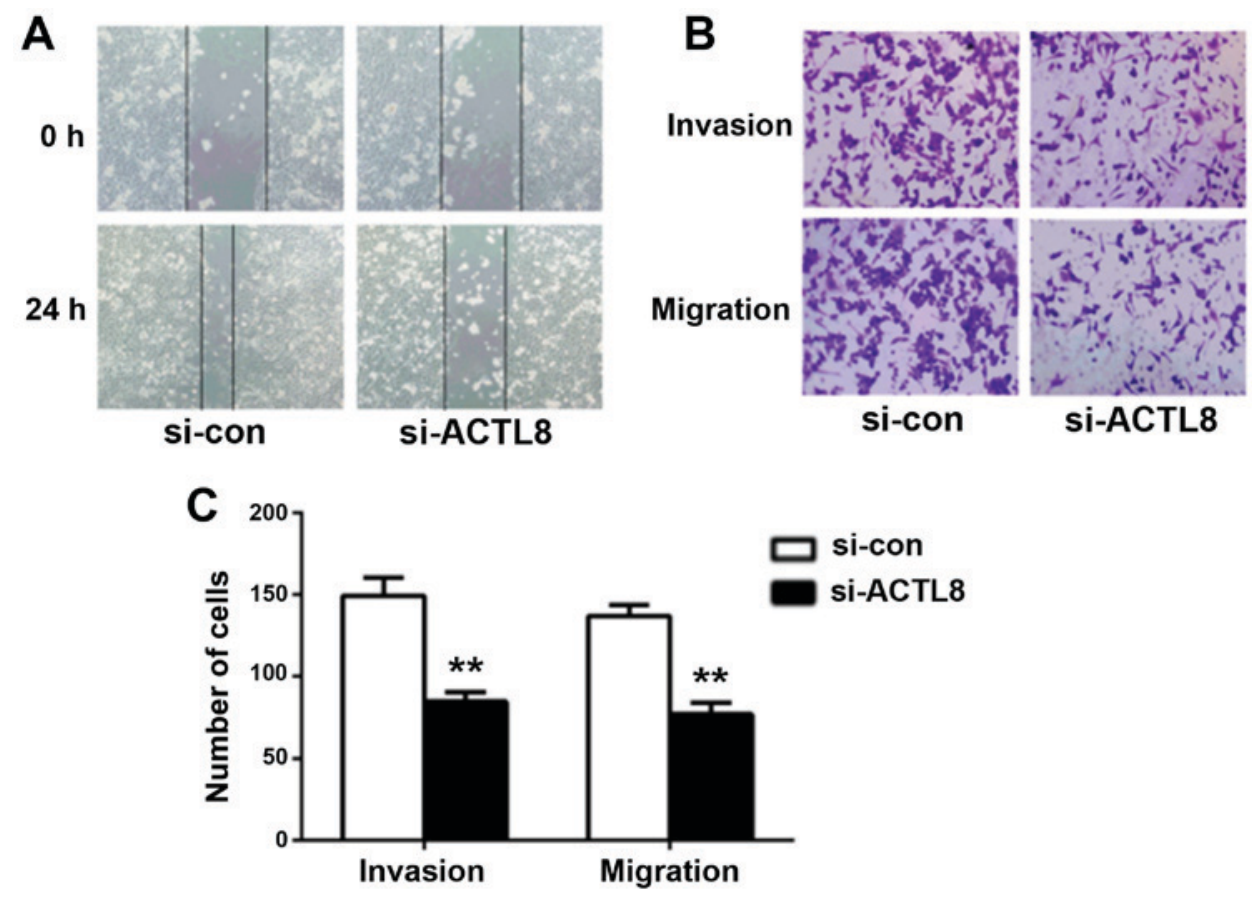

Figure 4. Knockdown of ACTL8 expression suppresses the invasion and migration of PCI-13 cells. (A) Representative microphotographs of wounding healing assay in PCI-13 cells were taken at 0 and $24 \mathrm{~h}$ following scratching. The wounding healing ability of PCI-13 cells in si-ACTL8 and si-con groups were compared $24 \mathrm{~h}$ after scratching. (B) Transwell chamber was used to detect the effect of ACTL8 knockout on the invasion and migration of PCI-13 cells. The number of invasive and migrated cells in si-ACTL8 group was evaluated by comparing with the si-con group. (C) The number of invasive and migrated cells was quantified compared with si-con group. Data were expressed as mean \pm standard deviation. ${ }^{* *} \mathrm{P}<0.01$ vs. si-con group. ACTL8, actin-like protein 8 ; si-ATCL8, small interfering RNA against ACTL8; HNSCC, head and neck squamous cell carcinoma; con, control.

Additionally, the expression level of ACTL8 mRNA and protein in si-ACTL8 group was significantly decreased by comparing the si-con group $(\mathrm{P}<0.01)$.

In addition, the effect of ACTL8 knockout on the proliferation of PCI-13 cells was further analyzed. A CCK-8 assay was used to measure the proliferative activity (Fig. 3C). Consequently, compared with the si-con group, the viability of PCI-13 in si-ACTL8 group was inhibited, inhibition reaching significantly levels at 72 and $96 \mathrm{~h}(\mathrm{P}<0.01)$. Furthermore, the results of colony formation assays in Fig. 3D demonstrated that the relative number of colonies for PCI-13 cells treated with ACTL8 siRNA was significantly reduced comparing with the si-con group $(\mathrm{P}<0.01)$, demonstrating that ACTL8 knockout inhibited the colony formation rate of PCI-13 cells. These results suggest that knockdown of ACTL8 contributes to inhibit the proliferation of PCI-13 cells.

ACTL8 knockout suppresses invasion and migration of PCI-13 cells. Considering the correlation between ACTL8 expression and lymph node metastasis in human HNSCC, it can be hypothesized that ACTL8 may serve a role in the invasion and migration of HNSCC cells. Therefore, wound healing and transwell assays were conducted to evaluate the effects of ACTL8 on HNSCC PCI-13 cells metastasis. Compared with the si-con group, the healing capacity for cells treated with si-ACTL8 following $24 \mathrm{~h}$ artificial scratching was decreased $(\mathrm{P}<0.05$; Fig. $4 \mathrm{~A})$. The results of the transwell assay in Fig. $4 \mathrm{~B}$ and $\mathrm{C}$ demonstrated that the numbers of invasive and migrated cells following interference with ACTL8 siRNA were significantly reduced compared with the si-con group $(\mathrm{P}<0.01)$. ACTL8 knockout efficiently decreased the invasion and migration capacity in HNSCC cells in vitro.

ACTL8 knockout inhibits the activation of the PI3K/AKT signaling pathway. The above studies indicate that ACTL8 suppressed the proliferative, invasive and migratory abilities of PCI-13 cells in HNSCC in vitro, whereas the underlying mechanisms remain unclear. The dysregulation of the PI3K/AKT signaling pathway is involved in a number of biological processes of various human diseases. PI3K/AKT signaling pathway has been reported to be activated in HNSCC and serve an important role in cell growth and survival (11). In the present study, western blotting assay was used to measure the expression of the key proteins in PI3K/AKT signaling pathway following transfection of ACTL8 siRNA into the HNSCC PCI-13 cell line. Western blot results in Fig. 5 revealed that ACTL8 knockout significantly decreased the levels of phosphorylated PI3K and AKT, as well as p70S6K in PCI-13 cells compared with the si-con group (all $\mathrm{P}<0.01$ ). Therefore, these results suggest that knockdown of ACTL8 may inhibit cell proliferation, invasion and migration by suppressing the activation of the PI3K/AKT signaling pathway in PCI-13 cells.

\section{Discussion}

HNSCC is one of the most common types of human cancer worldwide. It has the potential for early metastasis and is associated with a low survival rate (12). Currently, the prevention of locoregional tumor relapse is still a major challenge for the management of advanced HNSCC (13). Therefore, the investigation of novel prognostic biomarkers is of great importance 

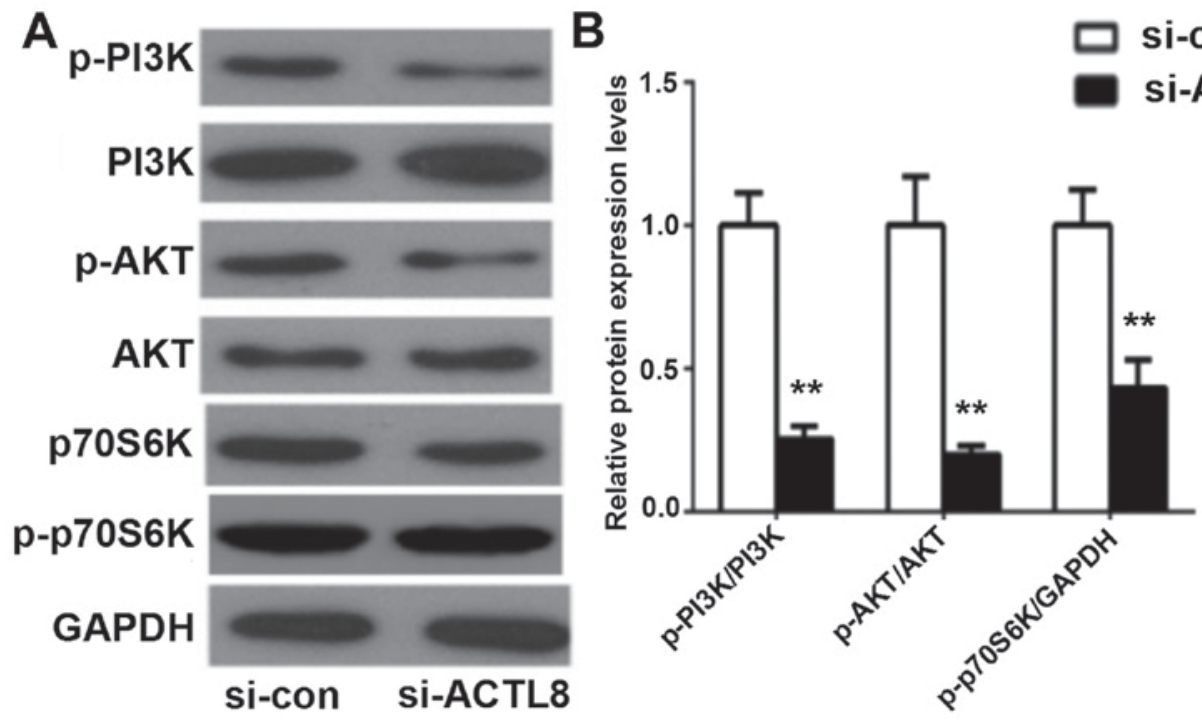

Figure 5. Knockdown of ACTL8 expression inhibits the activation of the PI3K/AKT signaling pathway. (A) Western blot assay was used to determine the expression levels of key proteins (PI3K, AKT and p70S6 K) in PI3K/AKT signaling pathway for cells treated by si-ACTL8 compared with the si-con group. (B) The expression levels of these proteins were quantified. The results are presented as the mean \pm standard deviation. ${ }^{* *} \mathrm{P}<0.01$ vs. si-con group. ACTL8, actin-like protein 8; si-con, small interfering control RNA; HNSCC, head and neck squamous cell carcinoma; AKT, protein kinase B; pPI3K, phosphorylated phosphoinositol 3 kinase; si-ATCL8, small interfering RNA against ACTL8.

for preventing tumor recurrence and metastasis and improving prognosis in patients with HNSCC (14).

The expression levels of CTA genes have been reported to be different in different tumor cells and the increased expression of CTA genes is an indicative of disease progression and poor prognosis $(15,16)$. Previously, CTA has been considered as targets of immune responses that were induced by cytotoxic $\mathrm{T}$ cells in certain tumors $(17,18)$. For example, members of CTA family MAGEA and NY-ESO-1/CTAG1B have been applied in clinical trials, including lung cancer and melanoma $(19,20)$. Additionally, Ipilimumab, the monoclonal antibody of ACTL4, has been used at the clinical trial phase (21). ACTL8, one of the CTA family has few studies in tumors. Freitas et al (7) noticed that ACTL8 is highly expressed in glioblastoma, but has no connection with the patient's survival. Additionally, Yao et al (8) studied the expression of the CTA gene ACTL8 in the basal subtype of breast cancer. However, the association between ACTL8 and tumorigenesis as well as prognosis in tumors remains unclear. Therefore, expression levels of ACTL8 in HNSCC were investigated in this study. The expression of ACTL8 in HNSCC was analyzed from the collected TCGA datasets and clinical tissue samples. The results indicated that ACTL8 in HNSCC tissues was highly expressed. Additionally, high expression in cells was also detected by RT-qPCR assay. Subsequently, the prognosis analysis was conducted, results demonstrated that the expression of ACTL8 in HNSCC could be an independent prediction factor and associated with the lymph node metastasis and tumor grade. Based on the siRNA strategy, a PCI-13 cell model with ACTL8 knockdown was established to analyze the functions of ACTL8 in HNSCC. As a result, due to the downregulation of ACTL8 expression in PCI-13 cells, the proliferation and colony formation rate of PCI-13 cells was decreased. In addition, the wound-healing assay and transwell method demonstrated that knockdown of ACTL8 suppressed the invasive and migratory capacity of PCI-13 cells. Therefore, all the above results suggest that
ACTL8 can be considered as a potential target for HNSCC therapy.

The PI3K/AKT signaling pathway is one of the most important intracellular signaling pathways, which has been demonstrated to be involved in the proliferation, angiogenesis, invasion and migration of tumor cells (22). It has been reported that alterations in crucial proteins of the PI3K/AKT signaling pathway have been involved in the progression of HNSCC (23). PI3K is an intracellular phosphatidylinositol kinase involved in various cellular functions, including cell proliferation, differentiation, apoptosis and growth $(24,25)$. AKT is an effector protein in the downstream of PI3K, which has been demonstrated to be important in carcinogenesis (26). In the present study, the expression levels of key proteins in PI3K/AKT signaling pathway were detected using a western blot assay. The results indicated that the expression levels of phosphorylated PI3K and AKT were reduced in PCI-13 cells transfected by ACTL8 siRNA. In addition, the downstream action target of the PI3K/AKT signaling pathway, p70S6K, serves a crucial role in translation initiation, cell metastasis, protein synthesis and cell cycle $(27,28)$. Similarly, the expression levels of p70S6 K in the PI3K/AKT signaling pathway were deceased following si-ACTL8 transfection in the present study. These results indicate that ACTL8 may be involved in HNSCC cell motility via the PI3K/AKT signaling pathway.

In conclusion, in the present study, ACTL8 was observed to be highly expressed in HNSCC and was associated with a poor prognosis. In addition, upon transfecting ACTL8 siRNA into HNSCC PCI-13 cells, the proliferative, invasive and migratory capacities of PCI-13 cells were inhibited, which may be associated with the inhibition of the activation of the PI3K/AKT signaling pathway. These results indicate that high expression of ACTL8 promotes the proliferation, invasion and migration of PCI-13 cells. Therefore, ACTL8 may be a potential prognosis marker and a novel therapeutic target for human HNSCC. 


\section{Acknowledgements}

Not applicable.

\section{Funding}

No funding was received.

\section{Availability of data and materials}

The datasets used and/or analyzed during the current study are available from the corresponding author on reasonable request.

\section{Authors' contributions}

BL and LM designed the study, and wrote and revised the manuscript. BL and JZ performed the study and analyzed the data. All authors read and approved the final manuscript.

\section{Ethics approval and consent to participate}

The present study obtained the informed consent of all patients and was approved by the Jinan Central Hospital Affiliated to Shandong University Medical Ethics Committee.

\section{Patient consent for publication}

The present study obtained the informed consent of all patients.

\section{Competing interests}

The authors declare they have no competing interests.

\section{References}

1. Agrawal N, Frederick MJ, Pickering CR, Bettegowda C, Chang K, Li RJ, Fakhry C, Xie TX, Zhang J, Wang J, et al: Exome sequencing of head and neck squamous cell carcinoma reveals inactivating mutations in NOTCH1. Science 333: 1154-1157, 2011.

2. Pries R, Nitsch S and Wollenberg B: Role of cytokines in head and neck squamous cell carcinoma. Expert Rev Anticancer Ther 6: 1195-1203, 2006.

3. Stacy DR, Ely K, Massion PP, Yarbrough WG, Hallahan DE, Sekhar KR and Freeman ML: Increased expression of nuclear factor E2 p45-related factor 2 (NRF2) in head and neck squamous cell carcinomas. Head Neck 28: 813-818, 2006.

4. Scanlan MJ, Simpson AJ and Old LJ: The cancer/testis genes: Review, standardization and commentary. Cancer Immun 4: 1, 2004.

5. Phuphanich S, Wheeler CJ, Rudnick JD, Mazer M, Wang H, Nuño MA, Richardson JE, Fan X, Ji J, Chu RM, et al: Phase I trial of a multi-epitope-pulsed dendritic cell vaccine for patients with newly diagnosed glioblastoma. Cancer Immunol Immunother 62: 125-135, 2013.

6. Atanackovic D, Blum I, Cao Y, Wenzel S, Bartels K, Faltz C, Hossfeld DK, Hegewisch-Becker S, Bokemeyer C and Leuwer R: Expression of cancer-testis antigens as possible targets for antigen-specific immunotherapy in head and neck squamous cell carcinoma. Cancer Biol Ther 5: 1218-1225, 2006.

7. Freitas M, Malheiros S, Stávale JN, Biassi TP, Zamunér FT, de Souza Begnami M, Soares FA and Vettore AL: Expression of cancer/testis antigens is correlated with improved survival in glioblastoma. Oncotarget 4: 636-646, 2013.

8. Yao J, Caballero OL, Yung WK, Weinstein JN, Riggins GJ, Strausberg RL and Zhao Q: Tumor subtype-specific cancer-testis antigens as potential biomarkers and immunotherapeutic targets for cancers. Cancer Immunol Res 2: 371-379, 2014.
9. Jin Y,Jin C, Wennerberg J,Mertens F and Höglund M: Cytogenetic and fluorescence in situ hybridization characterization of chromosome 1 rearrangements in head and neck carcinomas delineate a target region for deletions within 1p11-1p13. Cancer Res 58: 5859-5865, 1998.

10. Livak KJ and Schmittgen TD: Analysis of relative gene expression data using real-time quantitative PCR and the 2(-Delta Delta C(T)) method. Methods 25: 402-408, 2001.

11. Giudice FS and Squarize CH: The determinants of head and neck cancer: Unmasking the PI3K pathway mutations. J Carcinog Mutagen (Suppl 5): pii: 003, 2013.

12. Nohata N, Sone Y, Hanazawa T, Fuse M, Kikkawa N, Yoshino H, Chiyomaru T, Kawakami K, Enokida H, Nakagawa M, et al: miR-1 as a tumor suppressive microRNA targeting TAGLN2 in head and neck squamous cell carcinoma. Oncotarget 2: 29-42, 2011.

13. De Castro G, Dos Anjos CH, Lalami Y and Awada A: Clinical trial design in advanced head and neck cancer: From past experiences to future perspectives. Clin Invest 2: 473-481, 2012.

14. Le Tourneau C, Faivre S and Siu LL: Molecular targeted herapy of head and neck cancer: Review and clinical development challenges. Eur J Cancer 43: 2457-2466, 2007.

15. Dhodapkar MV, Osman K, Teruya-Feldstein J, Filippa D, Hedvat CV, Iversen K, Kolb D, Geller MD, Hassoun H, Kewalramani T, et al: Expression of cancer/testis (CT) antigens MAGE-A1, MAGE-A3, MAGE-A4, CT-7 and NY-ESO-1 in malignant gammopathies is heterogeneous and correlates with site, stage and risk status of disease. Cancer Immun 3: 9, 2003.

16. Balafoutas D, Zur HA, Mayer S, Hirschfeld M, Jaeger M, Denschlag D, Gitsch G, Jungbluth A and Stickeler E: Cancer testis antigens and NY-BR-1 expression in primary breast cancer: Prognostic and therapeutic implications. BMC Cancer 13: 271, 2013.

17. Taylor M, Bolton LM, Johnson P, Elliott T and Murray N: Breast cancer is a promising target for vaccination using cancer-testis antigens known to elicit immune responses. Breast Cancer Res 9: R46, 2007.

18. Atanackovic D, Arfsten J, Cao Y, Gnjatic S, Schnieders F, Bartels K, Schilling G, Faltz C, Wolschke C, Dierlamm J, et al: Cancer-testis antigens are commonly expressed in multiple myeloma and induce systemic immunity following allogeneic stem cell transplantation. Blood 109: 1103-1112, 2007.

19. Atanackovic D, Altorki NK, Cao Y, Ritter E, Ferrara CA, Ritter G, Hoffman EW, Bokemeyer C, Old LJ and Gnjatic S: Booster vaccination of cancer patients with MAGE-A3 protein reveals long-term immunological memory or tolerance depending on priming. Proc Natl Acad Sci USA 105: 1650-1655, 2008.

20. Fourcade J, Sun Z, Pagliano O, Chauvin JM, Sander C, Janjic B, Tarhini AA, Tawbi HA, Kirkwood JM, Moschos S, et al: PD-1 and Tim-3 regulate the expansion of tumor antigen-specific $\mathrm{CD} 8^{+} \mathrm{T}$ cells induced by melanoma vaccines. Cancer Res 74: 1045-1055, 2014.

21. Postow MA, Luke JJ, Bluth MJ, Ramaiya N, Panageas KS, Lawrence DP, Ibrahim N, Flaherty KT, Sullivan RJ, Ott PA, et al: Ipilimumab for patients with advanced mucosal melanoma. Oncologist 18: 726-732, 2013.

22. Britschgi A, Andraos R, Brinkhaus H, Klebba I, Romanet V, Müller U, Murakami M, Radimerski T and Bentires-Alj M: JAK2/STAT5 Inhibition circumvents resistance to PI3K/mTOR blockade: A rationale for cotargeting these pathways in metastatic breast cancer. Cancer Cell 22: 796-811, 2012.

23. Yang S, Ji Q, Chang B, Wang Y, Zhu Y, Li D, Huang C, Wang Y, Sun G, Zhang L, et al: STC2 promotes head and neck squamous cell carcinoma metastasis through modulating the PI3K/AKT/Snail signaling. Oncotarget 8: 5976-5991, 2017.

24. Zheng W, Nagaraju G, Liu Z and Liu K: Functional roles of the phosphatidylinositol 3-kinases (PI3Ks) signaling in the mammalian ovary. Mol Cell Endocrinol 356: 24-30, 2012.

25. Okkenhaug K and Vanhaesebroeck B: PI3K in lymphocyte development, differentiation and activation. Nat Rev Immunol 3: 317-330, 2003.

26. Yuan TL and Cantley LC: PI3K pathway alterations in cancer: Variations on a theme. Oncogene 27: 5497-5510, 2008.

27. Zhao XF and Gartenhaus RB: Phospho-p70S6K and cdc2/cdk1 as therapeutic targets for diffuse large B-cell lymphoma. Expert Opin Ther Targets 13: 1085-1093, 2009.

28. Wang F, Qiu Y, Zhang HM, Hanson P, Ye X, Zhao G, Xie R, Tong L and Yang D: Heat shock protein 70 promotes coxsackievirus B3 translation initiation and elongation via Akt-mTORC1 pathway depending on activation of p70S6K and Cdc2. Cell Microbiol, 2017.

This work is licensed under a Creative Commons Attribution-NonCommercial-NoDerivatives 4.0 International (CC BY-NC-ND 4.0) License. 